DOI 10.4171/JEMS/314

Olga Kharlampovich · Alexei Myasnikov

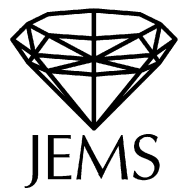

\title{
Limits of relatively hyperbolic groups and Lyndon's completions
}

Received September 9, 2009 and in revised form April 17, 2010

\begin{abstract}
We describe finitely generated groups $H$ universally equivalent (with constants from $G$ in the language) to a given torsion-free relatively hyperbolic group $G$ with free abelian parabolics. It turns out that, as in the free group case, the group $H$ embeds into Lyndon's completion $G^{\mathbb{Z}[t]}$ of the group $G$, or, equivalently, $H$ embeds into a group obtained from $G$ by finitely many extensions of centralizers. Conversely, every subgroup of $G^{\mathbb{Z}[t]}$ containing $G$ is universally equivalent to $G$. Since finitely generated groups universally equivalent to $G$ are precisely the finitely generated groups discriminated by $G$, the result above gives a description of finitely generated groups discriminated by $G$. Moreover, these groups are exactly the coordinate groups of irreducible algebraic sets over $G$.
\end{abstract}

\section{Introduction}

Denote by $\mathcal{G}$ the class of all non-abelian torsion-free relatively hyperbolic groups with free abelian parabolics. In this paper we describe finitely generated groups that have the same universal theory as a given group $G \in \mathcal{G}$ (with constants from $G$ in the language). We say that they are universally equivalent to $G$. These groups are central to the study of logic and algebraic geometry of $G$. It turns out that, as in the case when $G$ is a nonabelian free group [11], a finitely generated group $H$ universally equivalent to $G$ embeds into Lyndon's completion $G^{\mathbb{Z}[t]}$ of the group $G$, or equivalently, $H$ embeds into a group obtained from $G$ by finitely many extensions of centralizers. Conversely, every subgroup of $G^{\mathbb{Z}[t]}$ containing $G$ is universally equivalent to $G$ [2]. Let $H$ and $K$ be $G$-groups (contain $G$ as a subgroup). We say that a family of $G$-homomorphisms (homomorphisms identical on $G) \mathcal{F} \subset \operatorname{Hom}_{G}(H, K)$ separates [discriminates] $H$ into $K$ if for every nontrivial element $h \in H$ [every finite set $H_{0} \subset H$ of non-trivial elements] there exists $\phi \in \mathcal{F}$ such that $h^{\phi} \neq 1\left[h^{\phi} \neq 1\right.$ for every $\left.h \in H_{0}\right]$. In this case we say that $H$ is $G$-separated [G-discriminated] by $K$. Sometimes we do not mention $G$ and simply say that $H$ is separated [discriminated] by $K$. When $K$ is a free group we say that $H$ is freely separated [freely discriminated]. Since finitely generated groups universally equivalent to $G$ are precisely the finitely generated groups discriminated by $G$ ([1], [15]), the result above gives a description of finitely generated groups discriminated by $G$ or fully residually $G$-groups. These groups are exactly the coordinate groups of irreducible algebraic sets

O. Kharlampovich, A. Myasnikov: Department of Mathematics, McGill University, 805 Sherbrooke St. West, Montreal, QC, H3A 2K6 Canada; e-mail: okharlampovich@gmail.com, amiasnikov@gmail.com 
over $G$. Therefore we obtain a complete description of irreducible algebraic sets over $G$. Our proof uses the results of [7] and [17], [19].

\subsection{Algebraic sets}

Let $G$ be a group generated by $A$, and $F(X)$ the free group on $X=\left\{x_{1}, \ldots, x_{n}\right\}$. A system of equations $S(X, A)=1$ in variables $X$ and with coefficients from $G$ can be viewed as a subset of $G * F(X)$. A solution of $S(X, A)=1$ in $G$ is a tuple $\left(g_{1}, \ldots, g_{n}\right) \in G^{n}$ such that $S\left(g_{1}, \ldots, g_{n}\right)=1$ in $G$. The set $V_{G}(S)$ of all solutions of $S=1$ in $G$ is called the algebraic set defined by $S$.

The maximal subset $R(S) \subseteq G * F(X)$ with

$$
V_{G}(R(S))=V_{G}(S)
$$

is the radical of $S=1$ in $G$. The quotient group

$$
G_{R(S)}=G[X] / R(S)
$$

is the coordinate group of $S=1$.

The following conditions are equivalent:

- $G$ is equationally Noetherian, i.e., every system $S(X)=1$ over $G$ is equivalent to some finite part of itself;

- the Zariski topology (formed by algebraic sets as a subbasis of closed sets) over $G^{n}$ is Noetherian for every $n$, i.e., every proper descending chain of closed sets in $G^{n}$ is finite.

- every chain of proper epimorphisms of coordinate groups over $G$ is finite.

If the Zariski topology is Noetherian, then every algebraic set can be uniquely presented as a finite union of its irreducible components:

$$
V=V_{1} \cup \cdots \cup V_{k}
$$

Recall that a closed subset $V$ is irreducible if it is not the union of two proper closed subsets (in the induced topology).

\subsection{Fully residually $G$-groups}

A direct limit of a direct system of finite partial $n$-generated subgroups of $G$ such that all products of generators and their inverses eventually appear in these partial subgroups, is called a limit group over $G$. The same definition can be given using the notion of "marked group".

A marked group $(G, S)$ is a group $G$ with a prescribed family of generators $S=$ $\left(s_{1}, \ldots, s_{n}\right)$. Two marked groups $\left(G,\left(s_{1}, \ldots, s_{n}\right)\right)$ and $\left(G^{\prime},\left(s_{1}^{\prime}, \ldots, s_{n}^{\prime}\right)\right)$ are isomorphic as marked groups if the bijection $s_{i} \leftrightarrow s_{i}^{\prime}$ extends to an isomorphism. For example, $(\langle a\rangle,(1, a))$ and $(\langle a\rangle,(a, 1))$ are not isomorphic as marked groups. Denote by $\mathcal{G}_{n}$ the set of groups marked by $n$ elements up to isomorphism of marked groups. One can define a 
metric on $\mathcal{G}_{n}$ by setting the distance between two marked groups $(G, S)$ and $\left(G^{\prime}, S^{\prime}\right)$ to be $e^{-N}$ if they have exactly the same relations of length at most $N$. (This metric was used in [8], [5], [3].) Finally, a limit group over $G$ is a limit (with respect to the metric above) of marked groups $\left(H_{i}, S_{i}\right)$, where $H_{i} \leq G, i \in \mathbb{N}$, in $\mathcal{G}_{n}$.

The following two theorems summarize properties that are equivalent for a group $H$ to the property of being discriminated by $G$ (being $G$-discriminated by $G$ ).

Theorem A. [No coefficients] Let $G$ be an equationally Noetherian group. Then for a finitely generated group $H$ the following conditions are equivalent:

1. $\operatorname{Th}_{\forall}(G) \subseteq \operatorname{Th}_{\forall}(H)$, i.e., $H \in \mathbf{U} \mathbf{c l}(G)$;

2. $\operatorname{Th}_{\exists}(G) \supseteq \operatorname{Th}_{\exists}(H)$;

3. $H$ embeds into an ultrapower of $G$;

4. $H$ is discriminated by $G$;

5. $H$ is a limit group over $G$;

6. $H$ is defined by a complete atomic type in the theory $\operatorname{Th}_{\forall}(G)$;

7. $H$ is the coordinate group of an irreducible algebraic set over $G$ defined by a system of coefficient-free equations.

For a group $A$ we denote by $\mathcal{L}_{A}$ the language of groups with constants from $A$.

Theorem B. [With coefficients] Let $A$ be a group and $G$ an A-equationally Noetherian A-group. Then for a finitely generated A-group $H$ the following conditions are equivalent:

1. $\operatorname{Th}_{\forall, A}(G)=\mathrm{Th}_{\forall, A}(H)$;

2. $\mathrm{Th}_{\exists, A}(G)=\mathrm{Th}_{\exists, A}(H)$;

3. $H$ A-embeds into an ultrapower of $G$;

4. $H$ is A-discriminated by $G$;

5. $H$ is a limit group over $G$;

6. $H$ is a group defined by a complete atomic type in the theory $\operatorname{Th}_{\forall, A}(G)$ in the language $\mathcal{L}_{A}$;

7. $H$ is the coordinate group of an irreducible algebraic set over $G$ defined by a system of equations with coefficients in A.

Equivalences $1 \Leftrightarrow 2 \Leftrightarrow 3$ are standard results in mathematical logic. We refer the reader to [20] for the proof of $2 \Leftrightarrow 4$, to [9], [1] for the proof of $4 \Leftrightarrow 7$. Obviously, $2 \Rightarrow 5 \Rightarrow 3$. The above two theorems are proved in [4] for arbitrary equationally Noetherian algebras. Notice that in the case when $G$ is a free group and $H$ is finitely generated, $H$ is a limit group if and only if it is a limit group in the terminology of [21], [3] or [6], [7].

\subsection{Lyndon's completions of CSA-groups}

The paper [15], following Lyndon [14], introduced a $\mathbb{Z}[t]$-completion $G^{\mathbb{Z}[t]}$ of a given CSA-group $G$. In [2] it was shown that if $G$ is a CSA-group satisfying the Big Powers condition, then finitely generated subgroups of $G^{\mathbb{Z}[t]}$ are $G$-universally equivalent to $G$.

We refer to finitely generated $G$-subgroups of $G^{\mathbb{Z}[t]}$ as exponential extensions of $G$ (they are obtained from $G$ by iteratively adding $\mathbb{Z}[t]$-powers of group elements). The 
group $G^{\mathbb{Z}[t]}$ is the union of an ascending chain of extensions of centralizers of the group $G$ (see [15]).

A group obtained as the union of a chain of extensions of centralizers

$$
\Gamma=\Gamma_{0}<\Gamma_{1}<\cdots<\bigcup \Gamma_{k}
$$

where

$$
\Gamma_{i+1}=\left\langle\Gamma_{i}, t_{i} \mid\left[C_{\Gamma_{i}}\left(u_{i}\right), t_{i}\right]=1\right\rangle
$$

(extension of the centralizer $C_{\Gamma_{i}}\left(u_{i}\right)$ ) is called an iterated extension of centralizers and is denoted $\Gamma(U, T)$, where $U=\left\{u_{1}, \ldots, u_{k}\right\}$ and $T=\left\{t_{1}, \ldots, t_{k}\right\}$.

Every exponential extension $H$ of $G$ is also a subgroup of an iterated extension of centralizers of $G$.

\subsection{Relatively hyperbolic groups}

A group $G$ is hyperbolic relative to a collection $\left\{H_{\lambda}\right\}_{\lambda \in \Lambda}$ of subgroups (parabolic subgroups) if $G$ is finitely presented relative to $\left\{H_{\lambda}\right\}_{\lambda \in \Lambda}$,

$$
G=\left\langle X \cup\left(\mathcal{H}=\bigsqcup_{\lambda \in \Lambda} H_{\lambda}\right) \mid \mathcal{R}\right\rangle,
$$

and there is a constant $L>0$ such that for any word $W \in X \cup \mathcal{H}$ representing the identity in $G$ we have $\operatorname{Area}^{\mathrm{rel}}(W) \leq L\|W\|$, where $\operatorname{Area}^{\mathrm{rel}}(W)$ is the minimal number $k$ such that $W=\prod_{i=1}^{k} g_{i} R_{i} g_{i}^{-1}, r_{i} \in \mathcal{R}$, in the free product of the free group with basis $X$ and groups $\left\{H_{\lambda}\right\}_{\lambda \in \Lambda}$.

In [7, Theorem 5.16] Groves showed that groups from $\mathcal{G}$ are equationally Noetherian. By Theorem 1.14 of [17] the centralizer of every hyperbolic element from a group $G \in \mathcal{G}$ is cyclic. Therefore any non-cyclic abelian subgroup is contained in a finitely generated parabolic subgroup. It follows that finitely generated groups from $\mathcal{G}$ are CSA, that is, have malnormal maximal abelian subgroups (see also [6, Lemma 6.7]).

\subsection{Big Powers condition}

We say that an element $g \in G$ is hyperbolic if it is not conjugate to an element of one of the subgroups $H_{\lambda}, \lambda \in \Lambda$.

Proposition 1.1. Groups from $\mathcal{G}$ satisfy the Big Powers condition for hyperbolic elements: if $U$ is a set of hyperbolic elements, $g=g_{1} u_{1}^{n_{1}} g_{2} \ldots u_{k}^{n_{k}} g_{k+1}, u_{1}, \ldots, u_{k} \in U$, and $g_{i+1}^{-1} u_{i} g_{i+1}$ do not commute with $u_{i+1}$, then there exists a positive number $N$ such that if $\left|n_{i}\right| \geq N, i=1, \ldots, k$, then $g \neq 1$.

The proof of this proposition is similar to that of [19, Lemma 4.4] and was suggested by D. Osin.

The Cayley graph of $G$ with respect to the generating set $X \cup \mathcal{H}$ is denoted by $\Gamma(G, X \cup \mathcal{H})$. For a path $p$ in $\Gamma(G, X \cup \mathcal{H}), l(p)$ denotes its length, and $p_{-}$and $p_{+}$ denote the origin and the terminus of $p$, respectively. 
Definition 1.2 ([17]). Let $q$ be a path in the Cayley graph $\Gamma(G, X \cup \mathcal{H})$. A (non-trivial) subpath $p$ of $q$ is called an $H_{\lambda}$-component for some $\lambda \in \Lambda$ (or simply a component) if

(a) the label of $p$ is a word in the alphabet $H_{\lambda} \backslash\{1\}$;

(b) $p$ is not contained in a bigger subpath of $q$ satisfying (a).

Two $H_{\lambda}$-components $p_{1}, p_{2}$ of a path $q$ in $\Gamma(G, X \cup \mathcal{H})$ are called connected if there exists a path $c$ in $\Gamma(G, X \cup \mathcal{H})$ that connects some vertex of $p_{1}$ to some vertex of $p_{2}$ and the label of the path, denoted $\phi(c)$, is a word consisting of letters from $H_{\lambda} \backslash\{1\}$. In algebraic terms this means that all vertices of $p_{1}$ and $p_{2}$ belong to the same coset $g H_{\lambda}$ for a certain $g \in G$. Note that we can always assume that $c$ has length at most 1 , as every non-trivial element of $H_{\lambda} \backslash\{1\}$ is included in the set of generators. An $H_{\lambda}$-component $p$ of a path $q$ is called isolated (in $q$ ) if no distinct $H_{\lambda}$-component of $q$ is connected to $p$.

The following lemma can be found in [18, Lemma 2.7].

Lemma 1.3. Suppose that $G$ is a group hyperbolic relative to a collection $\left\{H_{\lambda} \mid \lambda \in \Lambda\right\}$ of subgroups. Then there exists a constant $K>0$ and finite subset $\Omega \subseteq G$ such that the following condition holds. Let $q$ be a cycle in $\Gamma(G, X \cup \mathcal{H}), p_{1}, \ldots, p_{k}$ a set of isolated components of $q$ for some $\lambda \in \Lambda$, and $g_{1}, \ldots, g_{k}$ the elements of $G$ represented by the labels of $p_{1}, \ldots, p_{k}$, respectively. Then for any $i=1, \ldots, k, g_{i}$ belongs to the subgroup $\langle\Omega\rangle \leq G$ and the word lengths of $g_{i}$ with respect to $\Omega$ satisfy the inequality

$$
\sum_{i=1}^{k}\left|g_{i}\right|_{\Omega} \leq K l(q)
$$

Recall also that a subgroup is elementary if it contains a cyclic subgroup of finite index. The lemma below is proved in [19].

Lemma 1.4. Let $g$ be a hyperbolic element of infinite order in $G$. Then

1. The element $g$ is contained in a unique maximal elementary subgroup $E_{G}(g)$ of $G$.

2. The group $G$ is hyperbolic relative to the collection $\left\{H_{\lambda} \mid \lambda \in \Lambda\right\} \cup\left\{E_{G}(g)\right\}$.

Proof of Proposition 1.1. It suffices to prove the proposition under the following additional assumption: if $u_{i}$ and $u_{j}$ are conjugate, then $u_{i}=u_{j}$, and if $u_{i}=u_{i+1}$, then $g_{i+1} \notin E\left(u_{i}\right)$. Indeed, if $u_{j}=h^{-1} u_{i} h$, we replace $u_{j}$ by $\bar{u}_{j}=u_{i}=h u_{j} h^{-1}, g_{j}$ by $\bar{g}_{j}=$ $g_{j} h^{-1}$ and $g_{j+1}$ by $\bar{g}_{j+1}=h g_{j+1}$. If $\left[g_{j}^{-1} u_{j-1} g_{j}, u_{j}\right] \neq 1$, then $h\left[g_{j}^{-1} u_{j-1} g_{j}, u_{j}\right] h^{-1}=$ $\left[\bar{g}_{j}^{-1} u_{j-1} \bar{g}_{j}, \bar{u}_{j}\right] \neq 1$. Similarly, if $\left[g_{j+1}^{-1} u_{j} g_{j+1}, u_{j+1}\right] \neq 1$, then $\left[\bar{g}_{j+1}^{-1} \bar{u}_{j} \bar{g}_{j+1}, u_{j+1}\right] \neq 1$. The CSA condition implies that $\left[g_{i+1}^{-1} u_{i} g_{i+1}, u_{i}\right]=1$ is equivalent to $g_{i+1} \in E\left(u_{i}\right)$.

Joining $g_{1}, \ldots, g_{k+1}$ to the finite relative generating set $X$ if necessary, we may assume that $g_{1}, \ldots, g_{k+1} \in X$. Set

$$
\mathcal{F}=\left\{\left.f \in\langle\Omega\rangle|| f\right|_{\Omega} \leq 4 K\right\},
$$

where $K$ and $\Omega$ are given by Lemma 1.3. Suppose that $g_{1} u_{1}^{n_{1}} \ldots g_{k} u_{k}^{n_{k}} g_{k+1}=1$. We consider a loop $p=q_{1} r_{1} q_{2} r_{2} \ldots q_{k} r_{k} q_{k+1}$ in $\Gamma(G, X \cup \mathcal{H})$, where $q_{i}$ (respectively, $\left.r_{i}\right)$ is labeled by $g_{i}$ (respectively by $u_{i}^{n_{i}}$ ). 


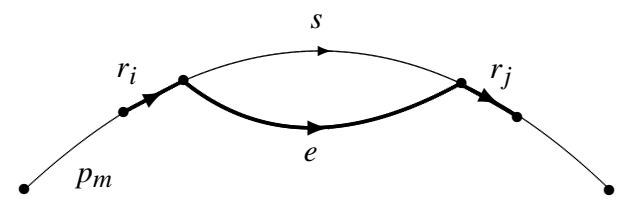

Fig. 1

Note that $r_{1}, \ldots, r_{k}$ are components of $p$. First assume that not all of these components are isolated in $p$. Suppose that $r_{i}$ is connected to $r_{j}$ for some $j>i$ and $j-i$ is minimal possible. Let $s$ denote the segment $\left[\left(r_{i}\right)_{+},\left(r_{j}\right)_{-}\right]$of $p$, and let $e$ be a path of length at most 1 in $\Gamma(G, X \cup \mathcal{H})$ labeled by an element of $H_{\lambda}$ such that $e_{-}=\left(r_{i}\right)_{+}$, $e_{+}=\left(r_{j}\right)_{-}$(see Fig. 1). If $j=i+1$, then the label $\operatorname{Lab}(s)$ is $g_{i+1}$. This contradicts the assumption $g_{i+1} \notin E\left(u_{i}\right)$ since $\operatorname{Lab}(s)$ and $\operatorname{Lab}(e)$ represent the same element in $G$. Therefore, $j=i+1+l$ for some $l \geq 1$. Note that the components $r_{i+1}, \ldots, r_{i+1+l}$ are isolated in the cycle $s e^{-1}$. (Indeed otherwise we can pass to another pair of connected components with smaller value of $j-i$.) By Lemma 1.3 we have $u_{q}^{n_{q}} \in\langle\Omega\rangle$ for all $i+1 \leq q \leq i+1+l$ and

$$
\sum_{q=i+1}^{i+l+1}\left|u_{q}^{n_{q}}\right|_{\Omega} \leq K l\left(s e^{-1}\right)=K(2 k+2) .
$$

Hence $\left|u_{p}^{n_{p}}\right|_{\Omega} \leq K(2+2 / k) \leq 4 K$ for at least one $p$, which is impossible for large $n_{p}$. Thus all components $r_{1}, \ldots, r_{k}$ are isolated in $p$. Applying now Lemma 1.3 again, we obtain

$$
\sum_{q=1}^{m}\left|u_{q}^{n_{q}}\right|_{\Omega} \leq K l(p)=K(2 k+2) .
$$

This is again impossible for large $n_{1}, \ldots, n_{k}$.

\subsection{Main results and the scheme of the proof}

Our main result is the following theorem.

Theorem C. [With constants] Let $\Gamma \in \mathcal{G}$. A finitely generated $\Gamma$-group $H$ is $\Gamma$-universally equivalent to $\Gamma$ if and only if $H$ is embeddable into $\Gamma^{\mathbb{Z}[t]}$.

The group $\Gamma^{\mathbb{Z}[t]}$ is discriminated by $\Gamma$. Indeed, it is enough to prove that any group $H$ obtained from $\Gamma$ by a finite series of extensions of centralizers is $\Gamma$-discriminated. We can obtain $H$ from $\Gamma$ in two steps. Let $K$ be a subgroup of $H$ that is obtained from $\Gamma$ by only extending centralizers of elements from parabolic subgroups. Then $K \in \mathcal{G}$ and $H$ is obtained from $K$ by a series of extensions of centralizers of hyperbolic elements. By Proposition 1.1 applied to each centralizer extension, $H$ is discriminated by $K$. Since $K$ is discriminated by $\Gamma$ by Lemma $1.3, H$ is also discriminated by $\Gamma$.

The proof of the converse follows the argument in [10], [11] with necessary modifications. It splits into steps. In Section 3 we will prove 
Theorem D. Let $\Gamma \in \mathcal{G}$ and $H$ a finitely generated group discriminated by $\Gamma$. Then $H$ embeds into an NTQ extension of $\Gamma$.

In Section 4 we will prove

Theorem E. Let $\Gamma \in \mathcal{G}$ and $\Gamma^{*}$ an NTQ extension of $\Gamma$. Then $\Gamma^{*}$ embeds into a group $\Gamma(U, T)$ obtained from $\Gamma$ by finitely many extensions of centralizers.

\section{Quadratic equations and NTQ systems and groups}

Definition 2.1. A standard quadratic equation over the group $G$ is an equation of one of the following forms (below $d, c_{i}$ are non-trivial elements from $G$ ):

$$
\begin{aligned}
\prod_{i=1}^{n}\left[x_{i}, y_{i}\right] & =1, \quad n>0 ; \\
\prod_{i=1}^{n}\left[x_{i}, y_{i}\right] \prod_{i=1}^{m} z_{i}^{-1} c_{i} z_{i} d & =1, \quad n, m \geq 0, m+n \geq 1 ; \\
\prod_{i=1}^{n} x_{i}^{2} & =1, \quad n>0 ; \\
\prod_{i=1}^{n} x_{i}^{2} \prod_{i=1}^{m} z_{i}^{-1} c_{i} z_{i} d & =1, \quad n, m \geq 0, n+m \geq 1 .
\end{aligned}
$$

Equations (1), (2) are called orientable of genus $n$, while equations (3), (4) are nonorientable of genus $n$.

Let $W$ be a strictly quadratic word over a group $G$. Then there is a $G$-automorphism $f \in \operatorname{Aut}_{G}(G[X])$ such that $W^{f}$ is a standard quadratic word over $G$.

To each quadratic equation one can associate a punctured surface. For example, the orientable surface associated to (2) will have genus $n$ and $m+1$ punctures.

Definition 2.2. Strictly quadratic words of the type $[x, y], x^{2}, z^{-1} c z$, where $c \in G$, are called atomic quadratic words or simply atoms.

By definition a standard quadratic equation $S=1$ over $G$ has the form

$$
r_{1} \ldots r_{k} d=1,
$$

where the $r_{i}$ are atoms and $d \in G$. The number $k$ is called the atomic rank of this equation; we denote it by $r(S)$.

Definition 2.3. Let $S=1$ be a standard quadratic equation written in the atomic form $r_{1} \ldots r_{k} d=1$ with $k \geq 2$. A solution $\phi: G_{R(S)} \rightarrow G$ of $S=1$ is called:

1. degenerate if $r_{i}^{\phi}=1$ for some $i$, and non-degenerate otherwise; 
2. commutative if $\left[r_{i}^{\phi}, r_{i+1}^{\phi}\right]=1$ for all $i=1, \ldots, k-1$, and non-commutative otherwise;

3. in general position if $\left[r_{i}^{\phi}, r_{i+1}^{\phi}\right] \neq 1$ for all $i=1, \ldots, k-1$.

Put

$$
\kappa(S)=|X|+\varepsilon(S),
$$

where $\varepsilon(S)=1$ if $S$ of the type (2) or (4), and $\varepsilon(S)=0$ otherwise.

Definition 2.4. Let $S=1$ be a standard quadratic equation over a group $G$ which has a solution in $G$. The equation $S(X)=1$ is regular if $\kappa(S) \geq 4$ (equivalently, the Euler characteristic of the corresponding punctured surface is at most -2 ) and there is a noncommutative solution of $S(X)=1$ in $G$, or it is an equation of the type $[x, y] d=1$ or $\left[x_{1}, y_{1}\right]\left[x_{2}, y_{2}\right]=1$.

Let $G$ be a group with a generating set $A$. A system of equations $S=1$ is called triangular quasi-quadratic (briefly, TQ) over $G$ if it can be partitioned into the following subsystems:

$$
\begin{aligned}
S_{1}\left(X_{1}, X_{2}, \ldots, X_{n}, A\right) & =1, \\
S_{2}\left(X_{2}, \ldots, X_{n}, A\right) & =1, \\
\ldots & \\
S_{n}\left(X_{n}, A\right) & =1,
\end{aligned}
$$

where for each $i$ one of the following holds:

1) $S_{i}$ is quadratic in the variables $X_{i}$;

2) $S_{i}=\left\{[y, z]=1,[y, u]=1 \mid y, z \in X_{i}\right\}$ where $u$ is a group word in $X_{i+1} \cup \cdots \cup$ $X_{n} \cup A$; in this case we say that $S_{i}=1$ corresponds to an extension of a centralizer;

3) $S_{i}=\left\{[y, z]=1 \mid y, z \in X_{i}\right\}$;

4) $S_{i}$ is the empty equation.

Sometimes, we join several consecutive subsystems $S_{i}=1, S_{i+1}=1, \ldots, S_{i+j}=1$ of a TQ system $S=1$ into one block, thus partitioning the system $S=1$ into new blocks. It is convenient to call a new system also a triangular quasi-quadratic system.

In the notation above define $G_{i}=G_{R\left(S_{i}, \ldots, S_{n}\right)}$ for $i=1, \ldots, n$ and put $G_{n+1}=G$. The TQ system $S=1$ is called non-degenerate (briefly, NTQ) if the following conditions hold:

5) each system $S_{i}=1$, where $X_{i+1}, \ldots, X_{n}$ are viewed as the corresponding constants from $G_{i+1}$ (under the canonical maps $X_{j} \rightarrow G_{i+1}, j=i+1, \ldots, n$ ), has a solution in $G_{i+1}$;

6) the element in $G_{i+1}$ represented by the word $u$ from 2) is not a proper power in $G_{i+1}$.

An NTQ system $S=1$ is called regular if each non-empty quadratic equation in $S_{i}$ is regular (see Definition 2.4). The coordinate group of an NTQ system (resp., a regular NTQ system) is called an NTQ group (resp., a regular NTQ group). 


\section{Embeddings into NTQ extensions}

Let $\Gamma \in \mathcal{G}$. In this section we will prove Theorem D. Namely, we will show how to embed a finitely generated fully residually $\Gamma$-group into an NTQ extension of $\Gamma$.

Theorem 3.1 ([7, Theorem 1.1]). Let $\Gamma \in \mathcal{G}$ and $G$ a finitely generated freely indecomposable group with abelian JSJ decomposition $\mathcal{D}$. Then there exists a finite collection $\left\{\eta_{i}: G \rightarrow L_{i}\right\}_{i=1}^{n}$ of proper quotients of $G$ such that, for any homomorphism $h: G \rightarrow \Gamma$ which is not equivalent to an injective homomorphism, there exists $h^{\prime}: G \rightarrow \Gamma$ with $h \sim h^{\prime}$ (the relation $\sim$ uses conjugation, canonical automorphisms corresponding to $\mathcal{D}$ and "bending moves"), $i \in\{1, \ldots, n\}$ and $h_{i}: L_{i} \rightarrow \Gamma$ so that $h^{\prime}=\eta_{i} h_{i}$. The quotient groups $L_{i}$ are fully residually $\Gamma$.

This theorem reduces the description of $\operatorname{Hom}(G, \Gamma)$ to a description of $\operatorname{Hom}\left(L_{i}, \Gamma\right)_{i=1}^{n}$. We then apply it again to each $L_{i}$ in turn and so on with successive proper quotients. Such a sequence terminates by equationally Noetherian property. Using this theorem one can construct a Hom-diagram which is the same as the so-called Makanin-Razborov diagram constructed in Section 6 of [7].

The statement of the above theorem is still true if we replace the set of all homomorphisms $h: G \rightarrow \Gamma$ by the set of all $\Gamma$-homomorphisms. The proof is the same. Therefore, a similar diagram can be constructed for $\Gamma$-homomorphisms $G \rightarrow \Gamma$.

Proof of Theorem D. Let $G$ be a finitely generated freely indecomposable group discriminated by $\Gamma$. According to the construction of the Makanin-Razborov diagram the set $\operatorname{Hom}(G, \Gamma)$ is divided into a finite number of families. Therefore one of these families contains a discriminating set of homomorphisms. Each family corresponds to a sequence of fully residually $\Gamma$-groups (see [13])

$$
G=G_{0}, G_{1}, \ldots, G_{n}
$$

where $G_{i+1}$ is a proper quotient of $G_{i}$ and $\pi_{i}: G_{i} \rightarrow G_{i+1}$ is an epimorphism. Similarly to Lemma 16 from [13], for a discriminating family, $\pi_{i}$ is a monomorphism for the following subgroups $H$ in the JSJ decomposition $\mathcal{D}_{i}$ of $G_{i}$ :

1. $H$ is a rigid subgroup in $\mathcal{D}_{i}$;

2. $H$ is an edge subgroup in $\mathcal{D}_{i}$;

3. $H$ is the subgroup of an abelian vertex group $A$ in $\mathcal{D}_{i}$ generated by the canonical images in $A$ of the edge groups of the edges of $\mathcal{D}_{i}$ adjacent to $A$.

We need the following result.

Lemma 3.2 ([13, Lemma 22]).

(1) Let $H=A *_{D} B, D$ be an abelian subgroup that is maximal abelian in $A$ or $B$, and $\pi: H \rightarrow \bar{H}$ be a homomorphism such that the restrictions of $\pi$ to $A$ and $B$ are injective. Put

$$
H^{*}=\left\langle\bar{H}, y \mid\left[C_{\bar{H}}(\pi(D)), y\right]=1\right\rangle .
$$


Then for every $u \in C_{H^{*}}\left((\pi(D)) \backslash C_{\bar{H}}(\pi(D))\right.$, the map

$$
\psi(x)= \begin{cases}\pi(x), & x \in A, \\ \pi(x)^{u}, & x \in B,\end{cases}
$$

gives rise to a monomorphism $\psi: H \rightarrow H^{*}$.

(2) Let $H=\left\langle A, t \mid d^{t}=c, d \in D\right\rangle$, where $D$ is abelian and either $D$ or its image is maximal abelian in $A$, and let $\pi: H \rightarrow \bar{H}$ be a homomorphism such that the restriction of $\pi$ to $A$ is injective. Put

$$
H^{*}=\left\langle\bar{H}, y \mid\left[C_{\bar{H}}(\pi(D)), y\right]=1\right\rangle .
$$

Then for every $u \in C_{H^{*}}\left((\pi(D)) \backslash C_{\bar{H}}(\pi(D))\right.$, the map

$$
\psi(x)= \begin{cases}\pi(x), & x \in A, \\ u \pi(x), & x=t,\end{cases}
$$

gives rise to a monomorphism $\psi: H \rightarrow H^{*}$.

Let now $\mathcal{D}$ be an abelian JSJ decomposition of $G$. Combining foldings and slidings, we can transform $\mathcal{D}$ into an abelian decomposition in which each vertex with non-cyclic abelian subgroup that is connected to some rigid vertex, is connected to only one vertex which is rigid. We suppose from the beginning that $\mathcal{D}$ has this property. Let $G_{1}$ be the fully residually $\Gamma$ proper quotient of $G$ on the next level of the Makanin-Razborov diagram, and $\pi$ be the canonical epimorphism $\pi: G \rightarrow G_{1}$. Let $G_{1}=P_{1} * \cdots * P_{\alpha} * F$ be the Grushko decomposition of $G_{1}$ relative to the set of all rigid subgroups and edge subgroups of $\mathcal{D}$. Here $F$ is the free factor and each $P_{i}$ is freely indecomposable modulo rigid subgroups and edge subgroups of $\mathcal{D}$.

We will construct a canonical extension $G^{*}$ of $\bar{G}=P_{1} * \cdots * P_{\alpha}$ which is the fundamental group of the graph of groups $\Lambda$ obtained from a single vertex $v$ with the associated vertex group $G_{v}=\bar{G}$ by adding finitely many edges corresponding to extensions of centralizers (viewed as amalgamated products) and finitely many $\mathrm{QH}$-vertices connected only to $v$. By construction of $\bar{G}$, each factor in this decomposition contains a conjugate of the image of some rigid subgroup or an edge group in $\mathcal{D}$. Indeed, the Grushko decomposition of $\bar{G}$ is non-trivial only if the fundamental groups of some separating simple closed curves on the surfaces corresponding to $\mathrm{QH}$ subgroups of $\mathcal{D}$ are mapped by $\pi$ to the identity element. Such curves cut the surface into pieces, and the fundamental groups of all the pieces that are not attached to rigid subgroups are mapped into $F$.

Let $g_{1}, \ldots, g_{l}$ be a fixed finite generating set of $\bar{G}$. For an edge $e \in \mathcal{D}$ we fix a tuple of generators $d_{e}$ of the abelian edge group $G_{e}$. The required extension $G^{*}$ of $\bar{G}$ is constructed in three steps. On each step we extend the centralizers $C_{\bar{G}}\left(\pi\left(d_{e}\right)\right)$ of some edges $e$ in $\mathcal{D}$ or add a $\mathrm{QH}$ subgroup. Simultaneously, to every edge $e \in \mathcal{D}$ we associate an element $s_{e} \in C_{G^{*}}\left(\pi\left(d_{e}\right)\right)$.

Step 1. Let $E_{\text {rig }}$ be the set of all edges between rigid subgroups in $\mathcal{D}$. One can define an equivalence relation $\sim$ on $E_{\text {rig }}$ by declaring for $e, f \in E_{\text {rig }}$ that

$$
e \sim f \Leftrightarrow \exists g_{e f} \in \bar{G}\left(g_{e f}^{-1} C_{\bar{G}}(\pi(e)) g_{e f}=C_{\bar{G}}(\pi(f))\right) .
$$


Let $E$ be a set of representatives of equivalence classes of $E_{\text {rig }}$ modulo $\sim$. We construct a group $G^{(1)}$ by extending every centralizer $C_{\bar{G}}\left(\pi\left(d_{e}\right)\right)$ of $\bar{G}, e \in E$, as follows. Let

$$
[e]=\left\{e=e_{1}, \ldots, e_{q_{e}}\right\}
$$

and $y_{e}^{(1)}, \ldots, y_{e}^{\left(q_{e}\right)}$ be new letters corresponding to the elements in $[e]$. Then put

$G^{(1)}$

$=\left\langle\bar{G}, y_{e}^{(1)}, \ldots, y_{e}^{\left(q_{e}\right)}(e \in E) \mid\left[C\left(\pi\left(d_{e}\right)\right), y_{e}^{(j)}\right]=1,\left[y_{e}^{(i)}, y_{e}^{(j)}\right]=1\left(i, j=1, \ldots, q_{e}\right)\right\rangle$.

One can associate with $G^{(1)}$ the system of equations over $\bar{G}$ :

$$
\left[\bar{g}_{e s}, y_{e}^{(j)}\right]=1, \quad\left[y_{e}^{(i)}, y_{e}^{(j)}\right]=1, \quad i, j=1, \ldots, q_{e}, s=1, \ldots, p_{e}, e \in E,
$$

where $y_{e}^{(j)}$ are new variables and the elements $\bar{g}_{e 1}, \ldots, \bar{g}_{e p_{e}}$ are constants from $\bar{G}$ which generate the centralizer $C\left(\pi\left(d_{e}\right)\right)$. We assume that the constants $\bar{g}_{e j}$ are given as words in the generators $g_{1}, \ldots, g_{l}$ of $\bar{G}$. We associate with the edge $e_{i} \in[e]$ an element $s_{e_{i}}$ that is the conjugate of $y_{e}^{(i)}$ from $C_{G^{(1)}}\left(\pi\left(d_{e_{i}}\right)\right)$.

Step 2. Let $A$ be a non-cyclic abelian vertex group in $\mathcal{D}$ and $A_{e}$ the subgroup of $A$ generated by the images in $A$ of the edge groups of edges adjacent to $A$. Then $A=\operatorname{Is}\left(A_{e}\right) \times A_{0}$ where $\operatorname{Is}\left(A_{e}\right)$ is the isolator of $A_{e}$ in $A$ (the minimal direct factor containing $\left.A_{e}\right)$ and $A_{0}$ a direct complement of $\operatorname{Is}\left(A_{e}\right)$ in $A$. Notice that the restriction of $\pi_{1}$ to $\operatorname{Is}\left(A_{e}\right)$ is a monomorphism (since $\pi_{1}$ is injective on $A_{e}$ and $A_{e}$ is of finite index in Is $\left(A_{e}\right)$ ). For each non-cyclic abelian vertex group $A$ in $\mathcal{D}$ we extend the centralizer of $\pi_{1}\left(\operatorname{Is}\left(A_{e}\right)\right)$ in $G^{(1)}$ by the abelian group $A_{0}$ and denote the resulting group by $G^{(2)}$. Observe that since $\pi_{1}\left(\operatorname{Is}\left(A_{e}\right)\right) \leq \bar{G}$ the group $G^{(2)}$ is obtained from $\bar{G}$ by extending finitely many centralizers of elements from $\bar{G}$.

If the abelian group $A_{0}$ has rank $r$ then the system of equations associated with the abelian vertex group $A$ has the form

$$
\left[y_{p}, y_{q}\right]=1, \quad\left[y_{p}, \bar{d}_{e j}\right]=1, \quad p, q=1, \ldots, r, j=1, \ldots, p_{e},
$$

where $y_{p}, y_{q}$ are new variables and the elements $\bar{d}_{e 1}, \ldots, \bar{d}_{e p_{e}}$ are constants from $\bar{G}$ which generate the subgroup $\pi\left(\operatorname{Is}\left(A_{e}\right)\right)$. We assume that the constants $\bar{d}_{e j}$ are given as words in the generators $g_{1}, \ldots, g_{l}$ of $\bar{G}$.

Step 3. Let $Q$ be a non-stable $\mathrm{QH}$ subgroup in $\mathcal{D}$ (not mapped by $\pi$ into the same $\mathrm{QH}$ subgroup). Suppose $Q$ is given by a presentation

$$
\prod_{i=1}^{n}\left[x_{i}, y_{i}\right] p_{1} \cdots p_{m}=1
$$

where there are exactly $m$ outgoing edges $e_{1}, \ldots, e_{m}$ from $Q$ and $\sigma\left(G_{e_{i}}\right)=\left\langle p_{i}\right\rangle, \tau\left(G_{e_{i}}\right)$ $=\left\langle c_{i}\right\rangle$ for each edge $e_{i}$. We add a $\mathrm{QH}$ vertex $Q$ to $G^{(2)}$ by introducing new generators and the quadratic relation

$$
\prod_{i=1}^{n}\left[x_{i}, y_{i}\right]\left(c_{1}^{\pi_{1}}\right)^{z_{1}} \cdots\left(c_{m-1}^{\pi_{1}}\right)^{z_{m-1}} c_{m}^{\pi_{1}}=1
$$


to the presentation of $G^{(2)}$. Observe that in the relations (7) the coefficients in the original quadratic relations for $Q$ in $\mathcal{D}$ are replaced by their images in $\bar{G}$.

Similarly, one introduces $\mathrm{QH}$ vertices for non-orientable $\mathrm{QH}$ subgroups in $\mathcal{D}$.

The resulting group is denoted by $G^{*}=G^{(3)}$.

We define a $(\Gamma)$-homomorphism $\psi: G \rightarrow G^{*}$ with respect to the splitting $\mathcal{D}$ of $G$ and will prove that it is a monomorphism. Let $T$ be the maximal subtree of $\mathcal{D}$. First, we define $\psi$ on the fundamental group of the graph of groups induced from $\mathcal{D}$ on $T$. Notice that if we consider only $\Gamma$-homomorphisms, then the subgroup $\Gamma$ is elliptic in $\mathcal{D}$, so there is a rigid vertex $v_{0} \in T$ such that $\Gamma \leq G_{v_{0}}$. The mapping $\pi$ embeds $G_{v_{0}}$ into $\bar{G}$, hence into $G^{*}$.

Let $P$ be a path $v_{0} \rightarrow v_{1} \rightarrow \cdots \rightarrow v_{n}$ in $T$ that starts at $v_{0}$. With each edge $e_{i}=$ $\left(v_{i-1} \rightarrow v_{i}\right)$ between two rigid vertex groups we have already associated the element $s_{e_{i}}$. Let us associate elements to other edges of $P$ :

a) if $v_{i-1}$ is a rigid vertex, and $v_{i}$ is either abelian or $\mathrm{QH}$, then $s_{e_{i}}=1$;

b) if $v_{i-1}$ is a $\mathrm{QH}$ vertex, $v_{i}$ is rigid or abelian, and the image of $e_{i}$ in the decomposition $\mathcal{D}^{*}$ of $G^{*}$ does not belong to $T^{*}$, then $s_{e_{i}}$ is the stable letter corresponding to the image of $e_{i}$;

c) if $v_{i-1}$ is a $\mathrm{QH}$ vertex and $v_{i}$ is rigid or abelian, and the image of $e_{i}$ in the decomposition of $G^{*}$ belongs to $T^{*}$, then $s_{e_{i}}=1$;

d) if $v_{i-1}$ is an abelian vertex with $G_{v_{i-1}}=A$ and $v_{i}$ is a $\mathrm{QH}$ vertex, then $s_{e_{i}}$ is an element from $A$ that belongs to $A_{0}$.

Since two abelian vertices cannot be connected by an edge in $\Gamma$, and we can suppose that two $\mathrm{QH}$ vertices are not connected by an edge, these are all possible cases.

We now define the embedding $\psi$ on the fundamental group corresponding to the path $P$ as follows:

$$
\psi(x)=\pi(x)^{S_{e_{i}} \ldots s_{e_{1}}} \quad \text { for } x \in G_{v_{i}} .
$$

This map is a monomorphism by Lemma 3.2. Similarly we define $\psi$ on the fundamental group of the graph of groups induced from $\mathcal{D}$ on $T$. We extend it to $G$ using the second statement of Lemma 3.2.

Recursively applying this procedure to $G_{1}$ and so on, we will construct the NTQ group $N$ such that $G$ is embedded into $N$. Theorem $\mathrm{D}$ is proved.

\section{Embedding of NTQ groups into $G(U, T)$}

An NTQ group $H$ over $\Gamma$ is obtained from $\Gamma$ by a series of extensions:

$$
\Gamma=H_{0}<H_{1}<\cdots<H_{n}=H,
$$

where for each $i=1, \ldots, n, H_{i}$ is either an extension of a centralizer in $H_{i-1}$ or the coordinate group of a regular quadratic equation over $H_{i-1}$. In the second case, equivalently, $H_{i}$ is the fundamental group of the graph of groups with two vertices, $v$ and $w$, such that $v$ is a $\mathrm{QH}$ vertex with $\mathrm{QH}$ subgroup $Q$, and $H_{i-1}$ is the vertex group of $w$. Moreover, there is a retraction from $H_{i}$ onto $H_{i-1}$. In this section we will prove the following theorem which, by induction, implies Theorem E. 
Theorem 4.1. Let $H$ be the fundamental group of the graph of groups with two vertices, $v$ and $w$, such that $v$ is a $Q H$ vertex with $Q H$ subgroup $Q, H_{w}=\Gamma \in \mathcal{G}$, and there is a retraction from $H$ onto $\Gamma$ such that $Q$ corresponds to a regular quadratic equation. Then $H$ can be embedded into a group obtained from $\Gamma$ by a series of extensions of centralizers.

The idea of the proof is as follows. Let $S_{Q}$ be a punctured surface corresponding to the $\mathrm{QH}$ vertex group in the decomposition $($ say $\mathcal{D})$ of $H$ as the graph of groups. We will find in Proposition 4.9 a finite collection of simple closed curves (s.c.c.) on $S_{Q}$ and a homomorphism $\delta: H \rightarrow K$, where $K$ is an iterated centralizer extension of $\Gamma * F$, with the following properties:

1) $\delta$ is a retraction on $\Gamma$,

2) each of the simple closed curves in the collection and all boundary elements of $S_{Q}$ are mapped by $\delta$ into non-trivial elements of $K$,

3) each connected component of the surface obtained by cutting $S_{Q}$ along this family of s.c.c. has Euler characteristic -1 ,

4) the fundamental group of each of these connected components is mapped monomorphically into a 2-generated free subgroup of $K$.

Given this collection of s.c.c. on the surface associated with the $\mathrm{QH}$ vertex group in the decomposition $\mathcal{D}$, one can extend $\mathcal{D}$ by further splitting the $\mathrm{QH}$ vertex groups along the family of simple closed curves described above. Now the statement of Theorem 4.1 will follow from Lemma 3.2.

Proposition 4.2 ([10, Prop. 3]). Let $S=1$ be a non-degenerate standard quadratic equation over a CSA-group $G$. Then either $S=1$ has a solution in general position, or every non-degenerate solution of $S=1$ is commutative.

Proving the theorem we will consider the following three cases for the equation corresponding to the $\mathrm{QH}$ subgroup $Q$ : orientable of genus $\geq 1$, genus $=0$, and non-orientable of genus $\geq 1$. For an orientable equation of genus $\geq 1$ we have the following proposition.

Proposition 4.3 (cf. [10, Prop. 4]). Let $S: \prod_{i=1}^{m}\left[x_{i}, y_{i}\right] \prod_{j=1}^{n} c_{j}^{z_{j}} g^{-1}=1$ ( $m \geq 1$, $n \geq 0)$ be a non-degenerate standard quadratic equation over a group $G \in \mathcal{G}$. Then $S=1$ has a solution in general position in some group $H$ which is an iterated extension of centralizers of $G * F$ (where $F$ is a free group) unless $S=1$ is the equation $\left[x_{1}, y_{1}\right]\left[x_{2}, y_{2}\right]=1$ or $[x, y] c^{z}=1$. This solution can be chosen so that the images of $x_{i}$ and $y_{i}$ generate a free subgroup (for each $\left.i=1, \ldots, m\right)$.

Proof. Let $n=0$. Then we have a standard quadratic equation of the type

$$
\left[x_{1}, y_{1}\right] \ldots\left[x_{k}, y_{k}\right]=g,
$$

which we will sometimes write as $r_{1} \ldots r_{k}=g$, where, as before, $r_{i}=\left[x_{i}, y_{i}\right]$.

Lemma 4.4. Let $S:\left[x_{1}, y_{1}\right]\left[x_{2}, y_{2}\right]=g$ be a non-degenerate equation over a group $G \in \mathcal{G}$. Then $S=g$ has a solution in general position in some group $H$ which is an iterated extension of centralizers of $G * F$ unless $S=1$ is the equation $\left[x_{1}, y_{1}\right]\left[x_{2}, y_{2}\right]=1$. Moreover, for each $i, x_{i}, y_{i}$ generate a free subgroup. 
Proof. Suppose $S=g$ has a solution $\phi$ such that $r_{1}^{\phi}=1$ and $r_{2}^{\phi}=1$. Then $g=1$ and our equation takes the form

$$
[x, y]\left[x_{2}, y_{2}\right]=1 \text {. }
$$

From now on we assume that for all solutions $\phi$ either $r_{1}^{\phi} \neq 1$ or $r_{2}^{\phi} \neq 1$.

Suppose now that just one of the equalities $r_{i}^{\phi}=1(i=1,2)$ holds, say $r_{1}^{\phi}=1$. Write $x_{2}^{\phi}=a$ and $y_{2}^{\phi}=b$. Then the equation is

$$
[x, y]\left[x_{2}, y_{2}\right]=[a, b] \neq 1 .
$$

This equation has other solutions, for example, for a new letter $c$ and $p>2$,

$$
\psi: x \rightarrow\left(c a^{-1}\right)^{-p} c, y \rightarrow c^{\left(c a^{-1}\right)^{p}}, x_{2} \rightarrow a^{\left(c a^{-1}\right)^{p}}, y_{2} \rightarrow\left(c a^{-1}\right)^{-p} b
$$

for which

$$
r_{1}^{\psi}=\left[c,\left(c a^{-1}\right)^{p}\right] \neq 1 \quad \text { and } \quad r_{2}^{\psi}=\left[\left(c a^{-1}\right)^{p}, a\right][a, b] \neq 1
$$

We claim that $\left[r_{1}^{\psi}, r_{2}^{\psi}\right] \neq 1$. Indeed, $\left[r_{1}^{\psi}, r_{2}^{\psi}\right]=1$ if and only if

$$
\left[\left[c,\left(c a^{-1}\right)^{p}\right],\left[\left(c a^{-1}\right)^{p}, a\right][a, b]\right]=1,
$$

but this is not true in $G *\langle c\rangle$.

Thus, just one case is left to consider. Suppose that $\left[r_{1}^{\phi}, r_{2}^{\phi}\right]=1$ and $r_{i}^{\phi} \neq 1(i=1,2)$ for all solutions $\phi$. Suppose $x^{\phi}=a, y^{\phi}=b, x_{2}^{\phi}=c$ and $y_{2}^{\phi}=d$. We will use ideas from [12] to change the solution. Let

$$
H=\left\langle G, t_{1}, t_{2}, t_{3}, t_{4}, t_{5} \mid 1=\left[t_{1}, b\right]=\left[t_{2}, t_{1} a\right]=\left[t_{3}, d\right]=\left[t_{4}, t_{3} c\right]=\left[t_{5}, t_{2} b c^{-1} t_{3}^{-1}\right]\right\rangle .
$$

Let $x^{\psi}=t_{5}^{-1} t_{1} a, y^{\psi}=\left(t_{2} b\right)^{t_{5}}, x_{2}=\left(t_{3} c\right)^{t_{5}}, y_{2}^{\psi}=t_{5}^{-1} t_{4} d$. This $\psi$ is also a solution of the same equation. But now $x^{\psi}$ and $y^{\psi}$ generate a free subgroup of $H$. If we have a word $w(x, y)$ then $w\left(x^{\psi}, y^{\psi}\right)=1$ in $H$ if all occurrences of $t_{5}$ disappear. This can only happen if $w(x, y)$ is made up of the blocks $x^{-1} y x$. But these blocks commute, hence $w=x^{-1} y^{n} x$. But now $w^{\psi}=a^{-1} t_{1}^{-1}\left(t_{2} b\right)^{n} t_{1} a$, therefore $w^{\psi}$ contains $t_{2}$ that does not disappear. Therefore $w^{\psi} \neq 1$. Similarly, $x_{2}^{\psi}$ and $y_{2}^{\psi}$ generate a free subgroup of $H$.

We will show now that $\left[r_{1}^{\psi}, r_{2}^{\psi}\right] \neq 1$. Indeed,

$$
r_{1}^{\psi} r_{2}^{\psi}=\left[x^{\psi}, y^{\psi}\right]\left[x_{2}^{\psi}, y_{2}^{\psi}\right]=[a, b][c, d]
$$

but

$$
r_{2}^{\psi} r_{1}^{\psi}=\left[x_{2}^{\psi}, y_{2}^{\psi}\right]\left[x^{\psi}, y^{\psi}\right]=t_{5}^{-1} c^{-1} t_{3}^{-1} t_{5} d^{-1} t_{3} c d a^{-1} t_{1}^{-1} b^{-1} t_{2}^{-1} t_{1} a t_{5}^{-1} t_{2} b t_{5} .
$$

And there is no way to make a pinch and cancel $t_{5}$ in the second expression. Therefore $\left[r_{1}^{\psi}, r_{2}^{\psi}\right] \neq 1$ and the proposition is proved.

Similarly, one can prove the following lemma. 
Lemma 4.5 (cf. $\left[10\right.$, Lemma 13]). Let $S:\left[x_{1}, y_{1}\right] \ldots\left[x_{k}, y_{k}\right]=g$ be a non-degenerate equation over a group $G \in \mathcal{G}$ and assume that $k \geq 3$. Then $S=g$ has a solution in general position over some group $H$ which is an iterated extension of centralizers of $G * F$. Moreover, for each $i, x_{i}, y_{i}$ generate a free subgroup.

Proof. The proof is by induction on $k$.

Let $k=3$. Assume that $g=1$. This means we have the equation

$$
\left[x_{1}, y_{1}\right]\left[x_{2}, y_{2}\right]\left[x_{3}, y_{3}\right]=1,
$$

which has a solution

$$
x_{1}^{\phi}=a, \quad y_{1}^{\phi}=b, \quad x_{2}^{\phi}=b, \quad y_{2}^{\phi}=a, \quad x_{3}^{\phi}=1, \quad y_{3}^{\phi}=1,
$$

where $a, b$ are arbitrary generators of $F$. Then the conclusion follows from Proposition 4 of [10]. But for convenience of the reader we will give a proof here. The equation

$$
\left[x_{2}, y_{2}\right]\left[x_{3}, y_{3}\right]=[b, a]
$$

is non-degenerate of atomic rank 2 ; hence, by the lemma above, it has a solution $\theta$ such that $\left[r_{2}^{\theta}, r_{3}^{\theta}\right] \neq 1$, and the images $x_{2}^{\theta}, y_{2}^{\theta}$ (resp., the images $x_{3}^{\theta}, y_{3}^{\theta}$ ) generate a free nonabelian subgroup. We got a solution $\psi$ such that

$$
x_{1}^{\psi}=a, \quad y_{1}^{\psi}=b, \quad x_{i}^{\psi}=x_{i}^{\theta}, \quad y_{i}^{\psi}=y_{i}^{\theta} \quad \text { for } i=2,3 .
$$

Now we are in a position to apply the previous lemma to the equation

$$
\left[x_{1}, y_{1}\right]\left[x_{2}, y_{2}\right]=\left[y_{3}^{\psi}, x_{3}^{\psi}\right] .
$$

It follows that there exists a solution to $S=g$ in general position and such that the subgroups generated by the images of $x_{i}, y_{i}$ are free non-abelian for $i=1,2,3$.

Assume now that $g \neq 1$. Then there exists a solution $\phi$ such that for at least one $i$ we have $r_{i}^{\phi} \neq 1$. Renaming variables one can assume that exactly $r_{3}^{\phi}=[a, b] \neq 1, a, b \in G$. Then the equation

$$
r_{1} r_{2}=g[b, a]
$$

has a solution in $G$. Again, we have two cases. If $g[b, a] \neq 1$, then we can argue as in Lemma 4.4. We obtain first a solution $\phi$ such that $x_{i}^{\phi}=c_{i}, y_{i}^{\phi}=d_{i}, i=1,2, x_{3}^{\phi}=$ $a, y_{3}^{\phi}=b,\left[r_{1}^{\phi}, r_{2}^{\phi}\right] \neq 1,\left[c_{1}, d_{1}\right] \neq g$, and $c_{i}, d_{i}$ generate a free subgroup for $i=1,2$. Then we consider the equation $\left[x_{2}, y_{2}\right]\left[x_{3}, y_{3}\right]=\left[d_{1}, c_{1}\right] g$ and apply Lemma 4.4 once more.

If $g[b, a]=1$ then $g=[a, b]$ and the initial equation $S=g$ actually has the form

$$
r_{1} r_{2} r_{3}=[a, b] .
$$

In this event consider a solution $\theta$ such that

$x_{1}^{\theta}=c, \quad y_{1}^{\theta}=d, \quad x_{2}^{\theta}=\left(c a^{-1}\right)^{-1} d, \quad y_{2}^{\theta}=c^{c a^{-1}}, \quad x_{3}^{\theta}=a^{c a^{-1}}, \quad y_{3}^{\theta}=\left(c a^{-1}\right)^{-1} b$, 
where $c, d$ are non-commuting elements from $F$. Then $\left[r_{i}^{\theta}, r_{j}^{\theta}\right] \neq 1, i, j=1,2,3$, and obviously $x_{i}^{\theta}, y_{i}^{\theta}$ generate a free group.

Let $k>3$. The equation

$$
r_{1} \ldots r_{k}=g
$$

has a solution $\phi$ such that for at least one $i$, say $i=k$ (by renaming variables we can always assume this), we have $r_{k}^{\phi}=[a, b] \neq 1$. Then the equation

$$
r_{1} \ldots r_{k-1}=g[b, a]
$$

is non-degenerate and by induction there is a solution $\theta$ such that $\left[r_{i}^{\theta}, r_{i+1}^{\theta}\right] \neq 1$ for all $i=1, \ldots, k-2$, and $x_{i}, y_{i}$ generate a free subgroup for $i=1, \ldots, k-1$. Define now a solution $\theta_{1}$ of the initial equation $S=g$ as follows:

$$
\begin{gathered}
x_{i}^{\theta}=x_{i}^{\theta_{1}}, \quad y_{i}^{\theta}=y_{i}^{\theta_{1}} \quad \text { for } i=1, \ldots, k-2, \\
x_{k-1}^{\theta_{1}}=t_{5}^{-1} t_{1} x_{k-1}^{\theta}, \quad y_{k-1}^{\theta_{1}}=\left(t_{2} y_{k-1}^{\theta}\right)^{t_{5}}, \quad x_{k}^{\theta_{1}}=\left(t_{3} a\right)^{t_{5}}, \quad y_{k}^{\theta_{1}}=t_{5}^{-1} t_{4} b,
\end{gathered}
$$

where

$$
\left[t_{1}, y_{k-1}^{\theta}\right]=\left[t_{2}, t_{1} x_{k-1}^{\theta}\right]=\left[t_{3}, b\right]=\left[t_{4}, t_{3} a\right]=\left[t_{5}, t_{2} y_{k-1}^{\theta} a^{-1} t_{3}^{-1}\right]=1 .
$$

This solution satisfies the requirements of the lemma.

Thus, Proposition 4.3 is proved for the case $n=0$. Consider now the case $n>0$.

Lemma 4.6 (cf. [10, Lemma 14]). The equation $S:[x, y] c^{z}=g$, where $g \neq 1$, which is consistent over a group $G \in \mathcal{G}$ always has a solution in general position in some iterated centralizer extension $H$ of $G$ such that the images of $x$ and $y$ generate a free subgroup.

Proof. Let $x \rightarrow a, y \rightarrow b, z \rightarrow d$ be an arbitrary solution of $[x, y] c^{z}=g$, where $g \neq 1$. Then $g=[a, b] c^{d}$ and the equation takes the form

$$
[x, y] c^{z}=[a, b] c^{d} .
$$

We can assume that $[a, b] \neq 1$. Indeed, suppose $[a, b]=1$. If $[c, d] \neq 1$, then we can write the equation as

$$
[x, y] c^{z}=c^{d}=\left[d, c^{-1}\right] c,
$$

which has the solution $x \rightarrow d, \quad y \rightarrow c^{-1}, \quad z \rightarrow 1$ such that $[x, y] \rightarrow\left[d, c^{-1}\right] \neq 1$. So we can assume now that $[c, d]=1$, in which case we have the equation

$$
[x, y] c^{z}=c \quad \text { or equivalently }[x, y]=\left[c^{-1}, z\right] .
$$

The group $G$ is a non-abelian CSA-group; hence the center of $G$ is trivial. In particular, there exists an element $h \in G$ such that $[c, h] \neq 1$. We see that $x \rightarrow c^{-1}, y \rightarrow h, z \rightarrow h$ is a solution $\phi$ for which $[x, y]^{\phi} \neq 1$.

Thus we have the equation $[x, y] c^{z}=[a, b] c^{d}$, where $[a, b] \neq 1$. Let $H=\langle G, t|$ $\left.\left[t, b c^{d}\right]=1\right\rangle$. Consider the map $\psi$ defined as follows:

$$
x^{\psi}=t^{-1} a, \quad y^{\psi}=t^{-1} b t, \quad z^{\psi}=d t .
$$


Straightforward computations show that

$$
[x, y]^{\psi}=[a, b][b, t], \quad\left(c^{z}\right)^{\psi}=c^{d t}
$$

hence

$$
\left[x^{\psi}, y^{\psi}\right] c^{z^{\psi}}=[a, b] c^{d},
$$

and consequently $\psi$ is a solution.

We claim that $\left[r_{1}^{\psi}, r_{2}^{\psi}\right] \neq 1$. Indeed, suppose $\left[r_{1}^{\psi}, r_{2}^{\psi}\right]=1$; then we have

$$
\begin{gathered}
{\left[[x, y]^{\psi}, c^{z^{\psi}}\right]=1, \quad\left[[a, b][b, t], c^{d t}\right]=1,} \\
t^{-1} b^{-1} t b[b, a] t^{-1} d^{-1} c^{-1} d t[a, b] b^{-1} t^{-1} b d^{-1} c d t=1,
\end{gathered}
$$

which implies

$$
t^{-1} b^{-1} t b[b, a] t^{-1} d^{-1} c^{-1} d t[a, b] b^{-1} b d^{-1} c d=1 .
$$

The letter $t$ disappears only if $c^{d}$ commutes with $b$ or $b^{a}$ commutes with $b c^{d}$. In both cases the last equality implies that $[a, b]$ commutes with $c^{d}$ and $b$ commutes with $b^{a}$. Therefore $[a, b]=1$, which contradicts the choice of $a, b, c, d$.

Now suppose that $m=1, n>1$. Let $\phi: G_{S} \rightarrow G$ be an arbitrary solution of $S=g$. Write

$$
h=g\left(\prod_{j=3}^{n} c_{j}^{z_{j}}\right)^{-\phi}
$$

and consider the equation

$$
[x, y] c_{1}^{z_{1}} c_{2}^{z_{2}}=h .
$$

If this equation satisfies the conclusion of Proposition 4.3, then by induction the equation $S=g$ will satisfy the conclusion. So we need to prove the proposition just for equation (10). There are now two possible cases.

Case (a): There exists a solution $\xi$ of the equation (10) such that $\left(c_{2}^{z_{2}}\right)^{\xi} \neq h$. In this event by Lemma 4.6 the equation

$$
[x, y] c_{1}^{z_{1}}=h\left(c_{2}^{z_{2}}\right)^{-\xi} \neq 1
$$

has a solution $\theta$ in general position. Hence we can extend this $\theta$ to a solution of (10) in such a way that $r_{i}^{\theta} \neq 1$ for $i=1,2$ and $\left[r_{1}^{\theta}, r_{2}^{\theta}\right] \neq 1$. Consequently, by Proposition 4.2 we can construct a solution $\psi$ in general position. It will automatically satisfy the conclusion of Proposition 4.3.

Case (b): Assume now that $\left(c_{2}^{z_{2}}\right)^{\phi}=h$ for all solutions $\phi$ of (10). Then we actually have

$$
[x, y] c_{1}^{z_{1}}=1, \quad c_{2}^{z_{2}}=h
$$

and this system of equations has a solution in $G$. It follows that $c_{1}=[a, b] \neq 1$ for some $a, b \in G$. Therefore equation (10) is

$$
[x, y][a, b]^{z_{1}} c_{2}^{z_{2}}=h,
$$


and has a solution $\psi$ of the type

$$
x^{\psi}=b^{f}, \quad y^{\psi}=a^{f}, \quad z_{1}^{\psi}=f, \quad z_{2}^{\psi}=z_{2}^{\phi}
$$

where $f$ is an arbitrary element in $G$ and $\phi$ is an arbitrary solution of (10). The two elements $[a, b]$ and $h$ are non-trivial in the CSA-group $G$, hence there exists $f^{*} \in G$ such that $\left[[a, b]^{f^{*}}, h\right] \neq 1$. But this implies that if we take $f=f^{*}$ then the solution $\psi$ will satisfy $\left[r_{2}^{\psi}, r_{3}^{\psi}\right] \neq 1$. Now it is sufficient to apply Proposition 4.2.

Now we suppose that $m=2, n>1$. Then we have the equation

$$
\left[x_{1}, y_{1}\right]\left[x_{2}, y_{2}\right] \prod_{j=1}^{n} c_{j}^{z_{j}}=g .
$$

Again, if there exists a solution $\phi$ of this equation such that

$$
\left(\prod_{j=1}^{n} c_{j}^{z_{j}}\right)^{\phi} \neq g
$$

then we can write

$$
h=g\left(\prod_{j=1}^{n} c_{j}^{z_{j}}\right)^{-\phi},
$$

and consider the equation

$$
\left[x_{1}, y_{1}\right]\left[x_{2}, y_{2}\right]=h
$$

which according to Lemma 4.5 has a solution $\xi$ in general position such that the images of $x_{i}, y_{i}$ generate a free subgroup. We can extend it to a solution of $S=g$, and by Proposition 4.3 applied to the equation

$$
\left[x_{1}^{\xi}, y_{1}^{\xi}\right]\left[x_{2}, y_{2}\right] \prod_{j=1}^{n} c_{j}^{z_{j}}=g
$$

we can construct a solution $\psi$ in general position with the required properties.

Assume now that

$$
\left(\prod_{j=1}^{n} c_{j}^{z_{j}}\right)^{\phi}=g
$$

for all solutions $\phi$ of the equation $S=g$. This implies that an arbitrary map of the type

$$
x_{1} \rightarrow a, \quad y_{1} \rightarrow b, \quad x_{2} \rightarrow b, \quad y_{2} \rightarrow a
$$

extends by means of any $\phi$ above to a solution $\psi$ of the equation $S=g$. Choose $a, b \in F$; then $\left[[b, a], r_{3}^{\phi}\right] \neq 1$ for the given solution $\phi$. And we again just need to appeal to Proposition 4.3 for the equation

$$
[a, b]\left[x_{2}, y_{2}\right] \prod_{j=1}^{n} c_{j}^{z_{j}}=g .
$$


The case $m>2$ is easy since if $\phi$ is a solution of the equation

$$
\prod_{i=1}^{m}\left[x_{i}, y_{i}\right] \prod_{j=1}^{n} c_{j}^{z_{j}} g^{-1}=1,
$$

then we can consider the equation

$$
\prod_{i=1}^{m}\left[x_{i}, y_{i}\right]=g\left(\prod_{j=1}^{n} c_{j}^{z_{j}}\right)^{-\phi},
$$

which by Lemma 4.5 has a solution in general position such that the images of $x_{i}, y_{i}$ generate a free subgroup; after that to finish the proof we need only apply Proposition 4.2.

Proposition 4.3 is proved.

The following proposition settles the genus 0 case.

Proposition 4.7. Let $S: c_{1}^{z_{1}} \ldots c_{k}^{z_{k}}=g$ be a non-degenerate standard quadratic equation over a group $G \in \mathcal{G}$. Then either $S=g$ has a solution in general position in some iterated centralizer extension of $G * F$, or every solution of $S=g$ is commutative.

Proof. By the definition of a standard quadratic equation, $c_{i} \neq 1$ for all $i=1, \ldots, k$. Hence every solution of $S=g$ is non-degenerate. Now the result follows from Proposition 4.2.

The following proposition can be proved similarly to Proposition 8 in [10].

Proposition 4.8. Let $S: x_{1}^{2} \ldots x_{p}^{2} c_{1}^{z_{1}} \ldots c_{k}^{z_{k}} g=1$ be a non-degenerate regular standard quadratic equation over a group $G \in \mathcal{G}$. Then there is a solution in general position in some iterated centralizer extension of $G * F$. If $p>2$ and $p+k>3$, then the equation is regular.

We now introduce some notation. For $S: \prod_{i=1}^{m}\left[x_{i}, y_{i}\right] \prod_{j=1}^{n} c_{j}^{z_{j}}=g$, denote $p_{j}=c_{j}^{z_{j}}$, $p_{n+1}=g^{-1}, q_{k}=\prod_{i=1}^{k}\left[x_{i}, y_{i}\right]$ for $k \leq m$ and $q_{m+k}=\prod_{i=1}^{m}\left[x_{i}, y_{i}\right] \prod_{j=1}^{k} p_{k}$.

For $S: \prod_{i=1}^{m} x_{i}^{2} \prod_{j=1}^{n} c_{j}^{z_{j}}=g$, denote $p_{j}=c_{j}^{z_{j}}, p_{n+1}=g^{-1}, q_{k}=\prod_{i=1}^{k} x_{i}^{2}$ for $k \leq m$ and $q_{m+k}=\prod_{i=1}^{m} x_{i}^{2} \prod_{j=1}^{k} p_{k}$.

Proposition 4.9. Let $S=g$ be a regular quadratic equation over a group $G \in \mathcal{G}$. Then there exists a solution $\delta$ in $G * F$ such that for any $j=1, \ldots, m+n-1$ :

1. $\left[q_{j}^{\delta}, r_{j+1}^{\delta}\right] \neq 1$;

2. $\left[q_{j}^{\delta},\left(r_{j+1} \ldots r_{n+m}\right)^{\delta}\right] \neq 1$;

3. there exists a solution $\delta$ in an iterated centralizer extension of $G * F$ such that the following subgroups are free non-abelian: $\left\langle q_{j}^{\delta}, r_{j+1}^{\delta}\right\rangle$ for any $j=1, \ldots, m+n-1$; $\left\langle q_{j}^{\delta}, x_{j+1}^{\delta}\right\rangle$ for any $j=1, \ldots, m-1 ;\left\langle q_{j+1}^{\delta}, x_{j+1}^{\delta}\right\rangle$ for any $j=1, \ldots, m-1$. 
Proof. Let $S=g$ be an orientable equation. We begin with the first statement. Let $\phi$ be a solution in general position constructed in Proposition 4.3. Let $q_{j-1}=\prod_{i=1}^{j-1}\left[x_{i}, y_{i}\right], A=$ $q_{j-1}^{\phi}, x_{j}^{\phi}=a, y_{j}^{\phi}=b, x_{j+1}^{\phi}=c, y_{j+1}^{\phi}=d$. If $[A[a, b],[c, d]] \neq 1$, then the statement is proved for $j$. Suppose that $[A[a, b],[c, d]]=1$. We can assume that $[b, c] \neq 1$ (taking $a b$ instead of $b$ if necessary). Let $t=b c^{-1}$. Take another solution $\psi$ such that $q_{j-1}^{\psi}=q_{j-1}^{\phi}$, $x_{j}^{\psi}=t^{-s} a, y_{j}^{\psi}=b^{t^{s}}, x_{j+1}^{\psi}=c^{t^{s}}, y_{j+1}^{\psi}=t^{-s} d$ for a large $s \in \mathbb{N}$.

If $\left[q_{j-1}^{\psi}\left[x_{j}^{\psi}, y_{j}^{\psi}\right],\left[x_{j+1}^{\psi}, y_{j+1}^{\psi}\right]\right]=1$, then

$$
A[a, b]\left[b, t^{s}\right]\left[t^{s}, c\right][c, d]=\left[t^{s}, c\right][c, d] A[a, b]\left[b, t^{s}\right],
$$

and therefore

$$
A[a, b][c, d]=\left[t^{s}, c\right] A[a, b][c, d]\left[b, t^{s}\right] .
$$

If we denote $B=A[a, b][c, d]$, this is equivalent to $B=\left[t^{s}, c\right] B\left[b, t^{s}\right]$, which is equivalent, by commutation transitivity, to $\left[t, c B b^{-1}\right]=1$ or $\left[t, B^{c^{-1}}\right]=1$, or $\left[B, c^{-1} b\right]=1$.

We take instead of $c, d$ respectively $\left(d^{p}\right) c,\left(\left(d^{p}\right) c\right)^{k} d$ and denote the new solution by $\delta_{s, p, k}$. If $\left[q_{j}^{\delta_{s, p, k}},\left[x_{j+1}^{\delta_{s, p, k}}, y_{j+1}^{\delta_{s, p, k}}\right]\right]=1$ for all $s, p, k$, then by the CSA property $\left[b\left(d^{p} c\right)^{-1},\left(d^{p} c\right)^{k} d\right]=1$ for all $p, k$, contrary to $c, d$ freely generating a free subgroup.

The proof for $j \geq m$ is similar.

The same solution $\delta_{s, p, k}$ can be used to prove the second statement.

We will now prove the third statement by induction on $j$. Let $\delta$ be a solution with properties 1 and 2 . Let $j=1$ and

$$
H_{1}=\left\langle G * F, t_{1} \mid\left[t_{1},\left(r_{2} \ldots r_{m+n}\right)^{\delta}\right]=1\right\rangle .
$$

We transform $\delta$ into a solution $\delta_{1}$ in the following way. If $m \neq 0$, then

$$
x_{1}^{\delta_{1}}=x_{1}^{\delta}, \quad y_{1}^{\delta_{1}}=y_{1}^{\delta}
$$

and

$$
x_{i}^{\delta_{1}}=x_{i}^{\delta t_{1}}, \quad y_{i}^{\delta_{1}}=y_{i}^{\delta t_{1}}, \quad z_{k}^{\delta_{1}}=z_{k}^{\delta} t_{1}
$$

for $i=2, \ldots, m, k=1, \ldots, n$. The subgroup generated by $q_{1}^{\delta_{1}}, r_{2}^{\delta_{1}}$ is free. Using Proposition 4.3 one can see that the subgroups generated by $q_{1}^{\delta_{1}}, x_{2}^{\delta_{1}}$ (if $m \geq 2$ ), and by $q_{2}^{\delta_{1}}, x_{2}^{\delta_{1}}$ are also free. In the case $m=0$ we define

$$
z_{1}^{\delta_{1}}=z_{1}^{\delta}, \quad z_{k}^{\delta_{1}}=z_{k}^{\delta} t_{1}
$$

for $i=2, \ldots, m, k=1, \ldots, n$.

Suppose by induction that a solution $\delta_{i-1}$ in a group $H_{j-1}$ which is an iterated centralizer extension of $G * F$ and satisfying the third statement of the proposition for indices from 1 to $j-1$ has been constructed. Let

$$
H_{j}=\left\langle H_{j-1}, t_{j} \mid\left[t_{j},\left(r_{j+1} \ldots r_{m+n}\right)^{\delta}\right]=1\right\rangle .
$$


We begin with the solution $\delta_{j-1}$ and transform it into a solution $\delta_{j}$ in the following way:

$$
\begin{aligned}
& x_{i}^{\delta_{j}}=x_{i}^{\delta_{j-1}}, \quad y_{i}^{\delta_{j}}=y_{i}^{\delta_{j-1}}, \quad i=1, \ldots, j, \\
& x_{i}^{\delta_{j}}=x_{i}^{\delta_{j-1} t_{j}}, \quad y_{i}^{\delta_{j}}=y_{i}^{\delta_{j-1} t_{j}}, \quad i=j+1, \ldots, m, \\
& z_{i}^{\delta_{j}}=z_{i}^{\delta_{j-1}} t_{j}
\end{aligned}
$$

The subgroups generated by $q_{j}^{\delta_{j}}, r_{j+1}^{\delta_{j}}$, by $q_{j}^{\delta_{j}}, x_{j+1}^{\delta_{j}}$ and by $q_{j+1}^{\delta_{j}}, x_{j+1}^{\delta_{j}}$ are free.

The proof for a non-orientable equation is very similar and we skip it.

We can now prove Theorem 4.1. Let $H$ be the fundamental group of the graph of groups with two vertices, $v$ and $w$, such that $v$ is a $\mathrm{QH}$ vertex, $H_{w}=\Gamma \in \mathcal{G}$, and there is a retraction from $H$ onto $\Gamma$. Let $S_{Q}$ be a punctured surface corresponding to a $\mathrm{QH}$ vertex group in this decomposition of $H$. Elements $q_{j}, x_{j}$ correspond to simple closed curves on the surface $S_{Q}$. By Proposition 4.9, we found a collection of simple closed curves on $S_{Q}$ and solution $\delta$ with a properties 1)-4) from the beginning of Section 4.

Theorem E now follows from Theorem 4.1 by induction.

Notice that Proposition 4.9 also implies the following

Corollary 4.10 (cf. [21, Lemma 1.32]). Let $Q$ be the fundamental group of a punctured surface $S_{Q}$ of Euler characteristic at most -2 . Let $\mu: Q \rightarrow \Gamma$ be a homomorphism that maps $Q$ into a non-abelian subgroup of $\Gamma$ and the image of every boundary component of $Q$ is non-trivial. Then either:

1. there exists a separating s.c.c. $\gamma \subset S_{Q}$ such that $\gamma$ is mapped non-trivially into $\Gamma$, and the image in $\Gamma$ of the fundamental group of each connected component obtained by cutting $S_{Q}$ along $\gamma$ is non-abelian, or

2. there exists a non-separating s.c.c. $\gamma \subset S_{Q}$ such that $\gamma$ is mapped non-trivially into $\Gamma$, and the image of the fundamental group of the connected component obtained by cutting $S_{Q}$ along $\gamma$ is non-abelian.

Acknowledgments. We thank D. Osin who suggested a proof of Proposition 1.1 and made other useful comments.

Both authors were supported by an NSERC grant.

\section{References}

[1] Baumslag, G., Myasnikov, A., Remeslennikov, V.: Algebraic geometry over groups I. Algebraic sets and ideal theory. J. Algebra 219, 16-79 (1999) Zbl 0938.20020 MR 1707663

[2] Baumslag, G., Myasnikov, A., Remeslennikov, V.: Discriminating completions of hyperbolic groups. Geom. Dedicata 92, 115-143 (2002) Zbl 1011.20041 MR 1934015

[3] Champetier, C., Guirardel, V.: Limit groups as limits of groups. Israel J. Math. 146, 1-75, Zbl 1103(2005)20026 MR 2151593

[4] Daniyarova, E., Myasnikov, A., Remeslennikov, V.: Unification theorems in algebraic geometry. In: Aspects of Infinite Groups, Algebra Discrete Math. 1, World Sci., 80-111 (2008) Zbl pre05526605 MR 2571513 
[5] Grigorchuk, R.: Degrees of growth of f.g. groups and the theory of invariant means. Izv. Akad. Nauk SSSR Ser. Mat. 48, 939-985 (1984) Zbl 0583.20023 MR 0764305

[6] Groves, D.: Limit groups for relatively hyperbolic groups I: The basic tools. Algebr. Geom. Topol. 9, 1423-1466 (2009) Zbl pre05597151 MR 2530123

[7] Groves, D.: Limit groups for relatively hyperbolic groups II: Makanin-Razborov diagrams. Geom. Topol. 9, 2319-2358 (2005) Zbl 1100.20032 MR 2209374

[8] Gromov, M.: Groups of polynomial growth and expanding maps. Publ. Math. IHES 53, 53-73 (1981) Zbl 0474.20018 MR 0623534

[9] Kharlampovich, O., Myasnikov, A.: Description of fully residually free groups and irreducible affine varieties over a free group. In: Summer School in Group Theory in Banff, 1996, CRM Proc. Lecture Notes 17, Amer. Math. Soc., 71-80 (1999) Zbl 0922.20027 MR 1653685

[10] Kharlampovich, O., Myasnikov, A.: Irreducible affine varieties over a free group. 1: irreducibility of quadratic equations and Nullstellensatz. J. Algebra 200, 472-516 (1998) Zbl 0904.20016 MR 1610660

[11] Kharlampovich, O., Myasnikov, A.: Irreducible affine varieties over a free group. II: Systems in triangular quasi-quadratic form and description of residually free groups. J. Algebra 200, 517-570 (1998) Zbl 0904.20017 MR 1610664

[12] Kharlampovich, O., Myasnikov, A.: Implicit function theorem over free groups. J. Algebra 290, 1-203 (2005) Zbl 1094.20016 MR 2154989

[13] Kharlampovich, O., Myasnikov, A.: Elementary theory of free non-abelian groups. J. Algebra 302, 451-552 (2006) Zbl 1110.03020 MR 2293770

[14] Lyndon, R. C.: Groups with parametric exponents. Trans. Amer. Math. Soc. 96, 518-533 (1960) Zbl 0108.02501 MR 0151502

[15] Myasnikov, A., Remeslennikov, V.: Exponential groups II: extensions of centralizers and tensor completion of CSA-groups. Int. J. Algebra Comput. 6, 687-711 (1996) Zbl 0866.20014 MR 1421886

[16] Myasnikov, A., Remeslennikov, V.: Algebraic geometry II: logical foundations. J. Algebra 234, 225-276 (2000) Zbl 0970.20017 MR 1799485

[17] Osin, D.: Relatively hyperbolic groups: intrinsic geometry, algebraic properties and algorithmic problems. Mem. Amer. Math. Soc. 179, no. 843 (2006) Zbl 1093.20025 MR 2182268

[18] Osin, D.: Peripheral fillings of relatively hyperbolic groups. Invent. Math. 167, 295-326 (2007) Zbl 1116.20031 MR 2270456

[19] Osin, D.: Elementary subgroups of hyperbolic groups and bounded generation. Int. J. Algebra Comput. 16, 99-118 (2006) Zbl 1100.20033 MR 2217644

[20] Remeslennikov, V.: E-free groups. Siberian Math. J. 30, 193-197 (1989) Zbl 0724.20025 MR 1043446

[21] Sela, Z.: Diophantine geometry over groups I: Makanin-Razborov diagrams. Publ. Math. IHES 93, 31-105 (2001) Zbl 1018.20034 MR 1863735 